\title{
Linfomas asociados a infección por Virus de Inmunodeficiencia Humana: Experiencia de un centro hospitalario de la $\mathrm{V}$ región, Chile
}

\author{
CHRISTINE ROJAS H. ${ }^{1}$, CARLOS MERINO M. ${ }^{1}$, \\ JUAN PABLO GHIRINGHELLI M. ${ }^{a}$, JOSÉ RAMÓN RODRÍGUEZ A. ${ }^{a}$, \\ FELIPE MARTÍNEZ L. ${ }^{1}$, WERNER JENSEN R. ${ }^{2}$
}

\section{Lymphomas associated with human immunodeficiency virus infection. Retrospective review of medical records}

Background: The incidence of lymphoma increases enormously in patients infected with the human immunodeficiency virus (HIV). Aim: To describe the incidence, clinical and histological characteristics, treatments and survival of lymphomas associated with HIV infection. Material and Methods: Retrospective review of medical records of patients with HIV and Lymphoma, treated in a public hospital, between January 2001 and June 2009. Results: Twenty-two male patients were included but 14 had immunohistochemical confirmation of the lymphoma. The accumulated incidence for this period was $2.8 \%$. The median age at lymphoma diagnosis was 39.5 years. Twelve patients (86\%) had non-Hodgkin Lymphoma (NHL) and two (14\%) Hodgkin Lymphoma. The main pathological type of non-Hodgkin lymphomas was diffuse large B cell in seven cases (50\%). The mean $\mathrm{CD}_{4}$ cell count and viral load were $83 \mathrm{cell} / \mathrm{mm}^{3}$ (33.5-113.5) and 26.000 RNA copies/ml (1210-196500), respectively. Twelve patients $(86 \%)$ had B type symptoms of lymphoma at the moment of diagnosis. Eleven patients (29\%) received chemotherapy with or without radiotherapy, one patient (7\%) received radiotherapy alone and two patients (14\%) received palliative symptomatic treatment. Six cases (43\%) received highly active antiretroviral therapy simultaneously with chemotherapy. Global mortality in this series was 57\% (8 patients) with a median survival time of 5.8 months (2.6-26.2). Conclusions: In this series of patients infected with HIV, a predominance of aggressive histological subtypes of lymphomas and low complete remission rates, were observed.

(Rev Med Chile 2011; 139: 27-35).

Key words: Anti-retroviral agents; HIV; Lymphoma.

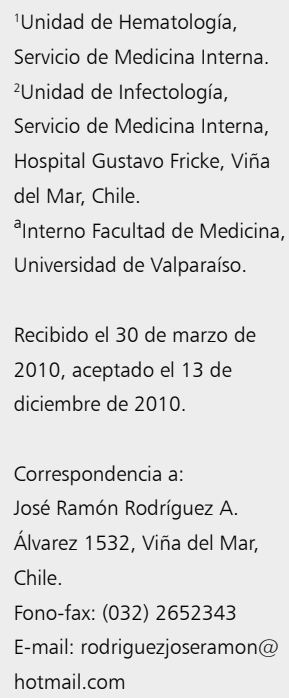

I a incidencia de linfoma no Hodgkin (LNH) se incrementa 60 a 200 veces en pacientes infectados por $\mathrm{VIH}^{1}$. La mayoría corresponden a linfomas de estirpe $\mathrm{B}$ agresivos, especialmente LNH difuso de células grandes B (DCGB) y LNH Burkitt. El linfoma Hodgkin (LH), en tanto, no se ha incluido como enfermedad diagnóstica de SIDA, aun cuando distintos estudios revelan una frecuencia 8-11 veces superior respecto a la población general ${ }^{1}$.

Tras la introducción de la terapia antirretroviral de gran actividad (TARGA), la incidencia de subtipos de LNH considerados condiciones definitorias de SIDA, se ha reducido de forma dra- 
mática ${ }^{2}$, determinando un ascenso en la incidencia de subtipos de linfoma de presentación habitual en población inmunocompetente ${ }^{3}$.

La evidencia disponible respecto a la asociación Linfoma/VIH, proviene mayoritariamente de estudios de cohorte desarrollados en Norteamérica, Europa y Africa $^{4-8}$. En Chile, existen escasos reportes acerca de la asociación de ambas patologías. Los registros de la Comisión Nacional del SIDA (CONASIDA) señalan una incidencia acumulada de 26 casos de LNH en 3.218 pacientes, hasta mayo de $2004^{9}$. Adicionalmente, dos series de pacientes, publicadas en 1995 y 2001, describen una incidencia de entre 1 y $1,7 \%^{10,11}$. El reporte más reciente, corresponde a una serie de 14 casos de linfoma en 2161 pacientes con diagnóstico de $\mathrm{VIH}$, con una incidencia acumulada de $0,68 \%{ }^{12}$.

Con el propósito de ahondar en el comportamiento y manejo de dicha asociación diagnóstica, se presenta la experiencia de un centro hospitalario del Servicio de Salud Viña del Mar-Quillota.

\section{Pacientes y Métodos}

Se realizó un estudio observacional retrospectivo, basado en la revisión de fichas clínicas del Policlínico de Infectología y Unidad de Hematología del Hospital Gustavo Fricke (HGF) de Viña del Mar.

Se incorporaron al estudio pacientes con diagnóstico de linfoma e infección por VIH, diagnosticados mediante estudio histológico e inmunohistoquímico, durante el período comprendido entre el 1 de enero de 2001 y el 1 de junio de 2009. Se excluyeron los casos sin confirmación histológica y aquellos con registros clínicos insuficientes.

El diagnóstico de infección por VIH fue confirmado en el Instituto de Salud Pública (ISP), mediante inmunofluorescencia indirecta (IFI). Se consideró como fecha de diagnóstico de $\mathrm{VIH}$, a la fecha de recepción del informe de confirmación del ISP. La confirmación mediante inmunohistoquímica se llevó a cabo en el Instituto de Anatomía Patológica. Se consideró como fecha de diagnóstico de linfoma a la fecha de recepción del informe anatomopatológico. Los antecedentes relativos a data y causa de muerte se obtuvieron a partir de certificados de defunción del Servicio de Registro Civil e Identificación.

La tipificación histológica de LNH y LH se efectuó de acuerdo a la clasificación de la Organización Mundial de la Salud para tumores de tejido linfoide ${ }^{1}$. Para efectos de etapificación clínica, se utilizaron los criterios de Ann Arbor ${ }^{13,14}$ y se calcularon los valores del Îndice Pronóstico Internacional (IPI) ${ }^{15}$ para LNH y Score Pronóstico de Hasenclever para LH en estadios avanzados (IPS) ${ }^{16}$.

Los antecedentes clínicos, antecedentes relativos a la infección por VIH y patología oncológica se registraron en un formulario elaborado para dicho propósito. Si existió fallecimiento se constató la fecha y causa de muerte y el tiempo de sobrevida desde el diagnóstico de linfoma.

La evaluación del estadio clínico y la respuesta terapéutica se efectuaron de acuerdo al protocolo del Programa Adulto Nacional de Drogas Antineoplásicas (PANDA) ${ }^{17}$ para linfomas. Se consideró remisión completa (RC) a la desaparición de cualquier evidencia de enfermedad al finalizar el tratamiento, remisión parcial (RP) a cualquier disminución de la masa tumoral sin alcanzar RC y no respuesta (NR) a la progresión de la enfermedad o ausencia de mejoría al finalizar el tratamiento.

El análisis estadístico se efectúo utilizando los programas SPSS 16 y Stata 10. Las variables cuantitativas se expresaron en medianas y rangos intercuartiles, mientras que las variables categóricas en frecuencias absolutas y porcentajes. Se generó una curva de sobrevida actuarial utilizando el método de Kaplan Meier. El cálculo de la incidencia anual e incidencia acumulada, se efectuó sobre el total de pacientes con diagnóstico histológico de linfoma. Se consideró como incidencia acumulada a la razón entre el número de casos nuevos de linfoma y el total de pacientes con VIH, durante el período en estudio. Para efectos de análisis descriptivo, solo se consideraron los pacientes confirmados mediante inmunohistoquímica. El estudio contó con la aprobación del Comité de Ética del Hospital Gustavo Fricke de Viña del Mar.

\section{Resultados}

A partir de 827 pacientes con diagnóstico de infección por VIH, se hallaron 26 casos con diagnóstico de linfoma. Se excluyeron cuatro pacientes, uno de ellos por contar con registros clínicos insuficientes y tres pacientes con sospecha clínica de LNH Primario del Sistema Nervioso Central sin confirmación histológica. 


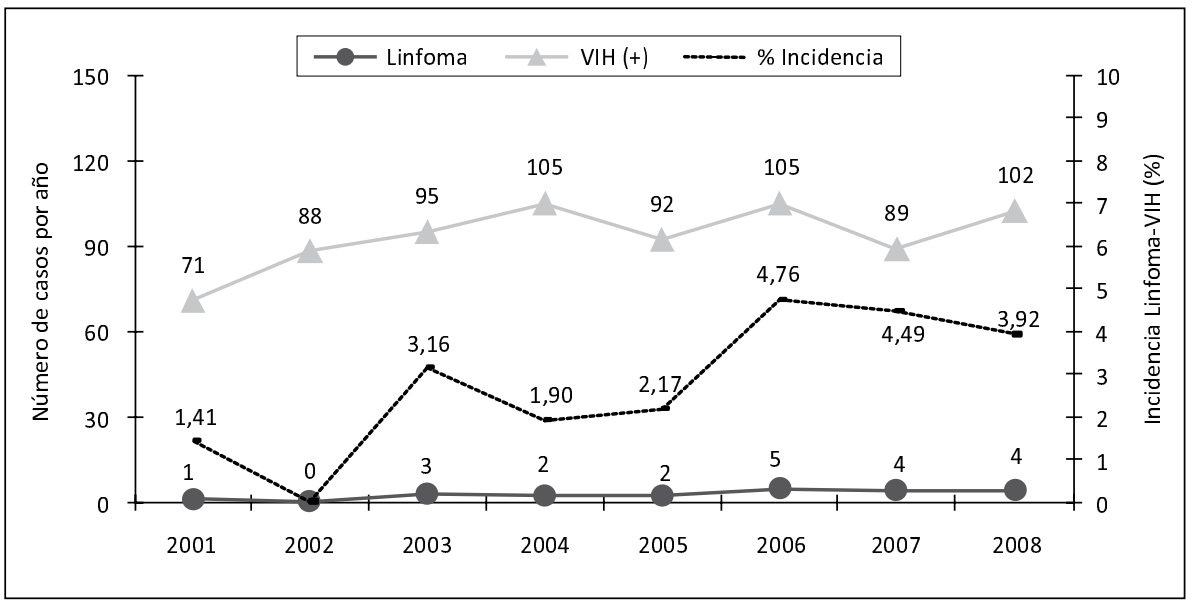

Figura 1. Número de casos nuevos de infección por $\mathrm{VIH}$ y Linfoma por año, e incidencia anual de Linfoma en pacientes con $\mathrm{VIH} / \mathrm{SIDA}$ en $\mathrm{HGF}$ 2001-2008.
En definitiva, se incluyeron 22 pacientes con diagnóstico histológico de linfoma. La incidencia acumulada de pacientes con linfoma y VIH, durante el período 2001-2008, fue de 2,81\%. En la Figura 1 se detalla el número de casos nuevos de infección por VIH y linfoma por año, e incidencia anual de esta asociación para el período en estudio.

Tabla 1. Características clínicas y demográficas de pacientes con linfoma y VIH $(n=14)$

\begin{tabular}{|lc|}
\hline Característica & \multicolumn{1}{c|}{$\begin{array}{c}\text { Frecuencia } \\
\text { (Porcentaje) }\end{array}$} \\
$\begin{array}{l}\text { Edad al diagnóstico Linfoma } \\
\text { (años)* }\end{array}$ & 39,5 \\
Edad al diagnóstico VIH & $(34-43,5)$ \\
(años)* & 35 \\
Género Masculino/Femenino & $(33-39)$ \\
Estadio clínico del linfoma & $14 / 0$ \\
I & \\
II & $4(28,5)$ \\
III & $2(14,2)$ \\
IV & $4(28,5)$ \\
Síntomas B del linfoma & $4(28,5)$ \\
Carga Viral (RNA/ml)* & $12(85,7)$ \\
& 26.000 \\
CD4 (céls/mm³)* & $(1.210-196.500)$ \\
& 83 \\
\hline
\end{tabular}

* Mediana-Rango intercuartil.
La confirmación con IHQ se efectuó en 14 de los 22 casos (63,6\%). Las características clínicas y demográficas para este grupo de pacientes se detallan en la Tabla 1. La edad mediana al diagnóstico de linfoma y VIH fue de 39,5 años $(34-43,5)$ y 35 años (33-39) respectivamente. Todos ellos fueron varones.

En cuatro de los 14 pacientes existió registro de la forma de contagio por VIH, siendo el contacto sexual la vía de transmisión común. La totalidad de los pacientes se encontraba en etapa SIDA al momento del diagnóstico de linfoma (Tabla 2). En ocho casos $(57,1 \%)$ el diagnóstico de linfoma precedió al de infección por VIH, confirmándose en un plazo de 1 a 3 meses (Mediana 39,5 días; 18-87,5) luego del diagnóstico de linfoma.

Referente a las manifestaciones clínicas que orientaron al diagnóstico de linfoma (Tabla 3), destaca la alta frecuencia de síntomas B del linfoma al diagnóstico (12/14 pacientes; $85,7 \%$ ) siendo la pérdida de peso el síntoma cardinal (11/14 pacientes; 78,5\%). La presencia de adenopatías constituyó el signo principal, con afectación multiganglionar en seis pacientes $(42,8 \%)$. Tres pacientes $(21,4 \%)$ debutaron con adenopatías de más de $10 \mathrm{~cm}$ de diámetro mayor (masa "bulky"). La presencia de hepato-esplenomegalia fue objetivada en sólo dos casos (14,2\%).

En la Tabla 4 se adjuntan la mediana de los principales exámenes de laboratorio. En seis casos $(42,8 \%)$, el recuento de linfocitos CD4 fue menor a 50 céls $/ \mathrm{mm}^{3}$. Las alteraciones inespecíficas del hemograma constituyeron un hallazgo frecuente, 
Tabla 2. Etapa CDC, enfermedad debut y características de la infección por VIH en pacientes con Linfoma y VIH

\begin{tabular}{|c|c|c|c|c|c|c|}
\hline \multirow[b]{2}{*}{$\begin{array}{l}\text { Paciente } \\
\text { no }^{\circ}\end{array}$} & \multirow[b]{2}{*}{$\begin{array}{l}\text { Etapa } \\
\text { CDC }\end{array}$} & \multirow[b]{2}{*}{$\begin{array}{l}\text { Enfermedad } \\
\text { debut }\end{array}$} & \multicolumn{4}{|c|}{ Características de la Infección VIH } \\
\hline & & & $\begin{array}{l}\text { Tiempo VIH (+) } \\
\text { al diagnóstico de } \\
\text { linfoma (meses) }\end{array}$ & $\begin{array}{c}\text { Carga Viral } \\
\text { (copias RNA/ml) }\end{array}$ & $\begin{array}{c}\text { CD4 } \\
\left(\text { cél } / \mathbf{m m}^{3}\right)\end{array}$ & $\begin{array}{c}\text { TARGA } \\
\text { Pre-Linfoma }\end{array}$ \\
\hline 1 & C3 & Hallazgo & 123 & 320.000 & 1 & No \\
\hline 2 & $\mathrm{C} 3$ & Linfoma & - & 230.000 & 43 & No \\
\hline 3 & $\mathrm{C3}$ & Neumonía $P$. jiroveci & 41 & $<80$ & 96 & Sí \\
\hline 4 & $\mathrm{C3}$ & $\mathrm{N} / \mathrm{D}$ & 192 & $\mathrm{~N} / \mathrm{D}$ & 100 & Sí \\
\hline 5 & $\mathrm{C3}$ & Linfoma & - & 4.600 & 17 & No \\
\hline 6 & $\mathrm{C} 2$ & Neurosífilis & 148 & $<80$ & 354 & Sí \\
\hline 7 & $\mathrm{C} 3$ & Linfoma & - & 74.000 & 104 & No \\
\hline 8 & $\mathrm{C} 3$ & Hallazgo & 40 & 92.000 & 142 & No \\
\hline 9 & $\mathrm{C} 3$ & Linfoma & - & 15.000 & 9 & No \\
\hline 10 & $\mathrm{C3}$ & Linfoma & - & $\mathrm{N} / \mathrm{D}$ & 39 & No \\
\hline 11 & $\mathrm{C} 3$ & Linfoma & - & 4.600 & 151 & No \\
\hline 12 & $\mathrm{C3}$ & Candidiasis esofágica & 5 & 520.000 & 47 & Sí \\
\hline 13 & $\mathrm{C3}$ & Linfoma & - & 37.000 & 73 & No \\
\hline 14 & $\mathrm{C} 3$ & Linfoma & - & $<80$ & 93 & No \\
\hline
\end{tabular}

TARGA: Terapia antirretroviral de gran actividad. N/D: No disponible.

Tabla 3. Manifestaciones clínicas al diagnóstico en pacientes con Linfoma y VIH $(n=14)$

\begin{tabular}{|lcc|}
\hline & $\begin{array}{c}\text { Frecuencia } \\
\text { (n) }\end{array}$ & $\begin{array}{c}\text { Porcentaje } \\
\text { (\%) }\end{array}$ \\
Síntomas & 1 & 7,1 \\
Artralgias/Mialgias & 2 & 14,3 \\
Aumento volumen local & 1 & 7,1 \\
Compromiso estado general & 1 & 7,1 \\
Disfagia lógica & 3 & 21,4 \\
Dolor abdominal & 11 & 78,6 \\
Pérdida de peso & 5 & 35,7 \\
Sensación febril & 2 & 14,3 \\
Síntomas neurológicos & 6 & 42,9 \\
Sudoración & 2 & 14,3 \\
Tos & & \\
Signos & 9 & 64,3 \\
Adenopatías & 8 & 57,1 \\
Fiebre & 1 & 7,1 \\
Focalidad neurológica & 4 & 28,6 \\
Tumoración & 7 & 50 \\
Compromiso extranodal & & \\
\hline
\end{tabular}

con presencia de anemia en doce pacientes $(85,7 \%)$, en rango entre 4,1 a $12,8 \mathrm{~g} / \mathrm{dl}$. El recuento diferencial de glóbulos blancos reveló neutropenia en tres pacientes $(21,4 \%)$ y linfopenia en siete casos (50\%). En diez casos $(71,4 \%)$ la VHS se elevó sobre su valor normal, siendo mayor a $100 \mathrm{~mm} / \mathrm{h}$ en seis pacientes $(42,8 \%)$. La $\beta-2$ microglobulinemia se cuantificó en sólo cinco casos, elevándose sobre su valor normal en cuatro de ellos $(28,5 \%)$.

El diagnóstico histológico se obtuvo mediante biopsia ganglionar en nueve pacientes $(64,2 \%)$, mientras que los casos restantes se diagnosticaron por biopsias extraganglionares (Tabla 5). En la mitad de los casos, se obtuvo biopsia de médula ósea, con evidencia de compromiso medular en tres pacientes $(21,4 \%)$.

En cuanto a las subtipos de linfoma, doce pacientes $(85,7 \%)$ correspondieron a LNH y dos $(14,2 \%)$ a LH, ambos de variedad celularidad mixta (Tabla 4). Para los LNH, los tipos histológicos más frecuentes fueron $\mathrm{LNH}$ DCGB (7/14 pacientes; 50\%) y LNH Burkitt (3/14 pacientes; $21,4 \%)$. 
Tabla 4. Valores de exámenes de laboratorio en pacientes con Linfoma y VIH

\begin{tabular}{|c|c|c|c|c|c|c|c|}
\hline $\begin{array}{l}H b(g / d l) \\
(n=14)\end{array}$ & $\begin{array}{c}\text { Leucocitos } \\
\left(\mathrm{cel} / \mathrm{mm}^{3}\right) \\
(\mathrm{n}=14)\end{array}$ & $\begin{array}{c}\text { Plaquetas } \\
\left.\text { (céls/mm } \mathbf{m m}^{3}\right) \\
(\mathbf{n}=14)\end{array}$ & $\begin{array}{c}\text { VHS } \\
(\mathrm{mm} / \mathrm{hr}) \\
(\mathrm{n}=14)\end{array}$ & $\begin{array}{c}\text { LDH (U/L) } \\
(n=14)\end{array}$ & $\begin{array}{c}\text { PCR } \\
(\mathrm{mg} / \mathrm{dl}) \\
(\mathrm{n}=14)\end{array}$ & $\begin{array}{c}\text { ß2 micro- } \\
\text { globulina } \\
(\mathrm{mg} / \mathrm{dl}) \\
(\mathrm{n}=5)\end{array}$ & $\begin{array}{c}\text { Bilirrubina } \\
\text { total } \\
(\mathbf{m g} / \mathrm{dl}) \\
(\mathrm{n}=14)\end{array}$ \\
\hline $\begin{array}{c}10 \\
(9-12,70)\end{array}$ & $\begin{array}{c}5.350 \\
(3.950-8.050)\end{array}$ & $\begin{array}{c}263.500 \\
(166.750-403.250)\end{array}$ & $\begin{array}{c}65,50 \\
(36,26-126,50)\end{array}$ & $\begin{array}{c}489,50 \\
(263-777,50)\end{array}$ & $\begin{array}{c}6,50 \\
(2,76-10)\end{array}$ & $\begin{array}{c}4,20 \\
(2,70-4,78)\end{array}$ & $\begin{array}{c}0,43 \\
(0,29-0,56)\end{array}$ \\
\hline
\end{tabular}

Tabla 5. Tipos de Linfoma, clasificación y tratamiento de pacientes con Linfoma y VIH

\begin{tabular}{|c|c|c|c|c|c|c|c|}
\hline $\begin{array}{l}\text { Paciente } \\
\text { n }\end{array}$ & $\begin{array}{l}\text { Tipo histológico. } \\
\text { Clasificación OMS y } \\
\text { sitio biopsia }\end{array}$ & $\begin{array}{l}\text { Estadio } \\
\text { clínico del } \\
\text { linfoma }\end{array}$ & IPI & Tratamiento & $\mathbf{R T}$ & $\begin{array}{c}\text { Condición } \\
\text { actual }\end{array}$ & $\begin{array}{c}\text { Sobrevida } \\
\text { (meses) }\end{array}$ \\
\hline 1 & $\begin{array}{l}\text { LNH Plasmablástico Cav. Oral } \\
\text { Biopsia: Mucosa oral }\end{array}$ & IV-B & 3 & $\mathrm{~N} / \mathrm{T}$ & $\mathrm{N} / \mathrm{T}$ & $\mathrm{F}$ & 0,80 \\
\hline 2 & $\begin{array}{l}\text { LNH tipo Burkitt } \\
\text { Biopsia: Ganglionar }\end{array}$ & III-B & 3 & $\mathrm{~N} / \mathrm{T}$ & $\mathrm{N} / \mathrm{T}$ & $\mathrm{F}$ & 0,83 \\
\hline 3 & $\begin{array}{l}\text { LNH DCGB } \\
\text { Biopsia: Tumor Maxilar Inferior }\end{array}$ & $\mathrm{I}-\mathrm{B}$ & 2 & $\mathrm{CHOP}$ & NR & $\mathrm{F}$ & 5,10 \\
\hline 4 & $\begin{array}{l}\text { LNH DCGB } \\
\text { Variante anaplásica } \\
\text { Biopsia: Ganglionar }\end{array}$ & III-B & 2 & Rt & NR & $\mathrm{F}$ & 3,00 \\
\hline 5 & $\begin{array}{l}\text { LNH DCGB variante anaplásica } \\
\text { Biopsia: Ganglionar }\end{array}$ & IV-BS & 2 & $\begin{array}{l}\text { CHOP/ } \\
\text { Intratecal }\end{array}$ & $\mathrm{RC}$ & V & 6,47 \\
\hline 6 & $\begin{array}{l}\text { LNH tipo Burkitt } \\
\text { Biopsia: Glándula parótida }\end{array}$ & IE-A & 2 & $\begin{array}{l}\text { CHOP/ } \\
\text { Intratecal }\end{array}$ & $\mathrm{RP}$ & $\mathrm{F}$ & 5,17 \\
\hline 7 & $\begin{array}{l}\text { LNH Plasmablástico Cav. Oral } \\
\text { Biopsia: Mucosa oral }\end{array}$ & $\mathrm{I}-\mathrm{B}$ & 1 & $\begin{array}{l}\text { CHOP/ } \\
\text { Intratecal }\end{array}$ & $\mathrm{RC}$ & V & 29,37 \\
\hline 8 & $\begin{array}{l}\text { LH Celularidad Mixta } \\
\text { Biopsia: Ganglionar }\end{array}$ & III-B & - & ABVD/Rt & $\mathrm{RC}$ & V & 30,47 \\
\hline 9 & $\begin{array}{l}\text { LNH DCGB Tipo Centro Germinal } \\
\text { Biopsia: Ganglionar }\end{array}$ & III-B & 2 & $\mathrm{CHOP}$ & $\mathrm{RC}$ & V & 1,33 \\
\hline 10 & $\begin{array}{l}\text { LNH DCGB Tipo Centroblástico } \\
\text { Biopsia: Mucosa oral }\end{array}$ & IV-B & 3 & EPOCH & NR & $\mathrm{F}$ & 7,30 \\
\hline 11 & $\begin{array}{l}\text { LNH DCGB Tipo Centroblástico } \\
\text { Biopsia: Ganglionar }\end{array}$ & $\mathrm{I}-\mathrm{B}$ & 1 & $\mathrm{CHOP} / \mathrm{Rt}$ & $\mathrm{RC}$ & $\mathrm{F}$ & 27,27 \\
\hline 12 & $\begin{array}{l}\text { LNH tipo Burkitt } \\
\text { Biopsia: Ganglionar }\end{array}$ & IV-A & 3 & $\begin{array}{l}\text { CHOP/ } \\
\text { Intratecal }\end{array}$ & $\mathrm{RP}$ & V & 17,17 \\
\hline 13 & $\begin{array}{l}\text { LNH DCGB tipo Centroblástico } \\
\text { Biopsia: Ganglionar }\end{array}$ & II-B & 1 & $\mathrm{CHOP}$ & $\mathrm{RP}$ & $\mathrm{F}$ & 4,40 \\
\hline 14 & $\begin{array}{l}\text { LH Celularidad mixta } \\
\text { Biopsia: Ganglionar }\end{array}$ & II-B & - & $A B V D$ & $\mathrm{RC}$ & V & 25,83 \\
\hline
\end{tabular}

IPI: Indice Pronóstico Internacional. RC: Remisión completa. F: Fallecimiento. NR: No respuesta. RP: Remisión parcial. V: Vivo. RT: Respuesta terapéutica. N/T: No tratado. Rt: Radioterapia. 
Ocho pacientes $(57,1 \%)$ se encontraban en estadios avanzados del linfoma (III-IV) al momento del diagnóstico. De acuerdo al puntaje IPI, seis pacientes fueron clasificados como casos de bajo riesgo, cuatro de riesgo intermedio-bajo y tres de riesgo intermedio-alto. En tanto que el puntaje de IPS para el único caso de LH avanzado de esta serie fue de 1 punto.

La conducta terapéutica, incluyendo tipo de tratamiento utilizado y respuesta terapéutica, se detalla en la Tabla 5. Dos pacientes (14,2\%) no fueron tratados por hallarse fuera del alcance terapéutico, falleciendo de forma precoz luego del diagnóstico. Once pacientes $(78,5 \%)$ recibieron tratamiento con quimioterapia asociado o no a radioterapia y un paciente $(7,1 \%)$ fue tratado con radioterapia exclusiva. Seis pacientes $(42,8 \%)$ recibieron TARGA de forma concomitante a la quimioterapia. Dos casos $(14,2 \%)$ ya habían recibido quimioterapia por otro linfoma previo al período en estudio, uno de ellos correspondió a LH celularidad mixta tratado con radioterapia exclusiva, y otro fue un LNH de células del manto, tratado con 7 ciclos de quimioterapia COP (ciclofosfamida, vincristina y prednisona).

Los esquemas de quimioterapia utilizados para LNH fueron CHOP (ciclofosfamida, doxorrubicina, vincristina, prednisona) y $\mathrm{EPOCH}$ (etopósido, prednisona, vincristina, ciclofosfamida, doxorrubicina). En LH se empleó el esquema ABVD (doxorrubucina, bleomicina, vinblastina, dacarbazina). Ningún paciente recibió tratamiento con rituximab.

Respecto a la respuesta terapéutica, seis pacientes $(42,8 \%)$ presentaron $R C$, tres pacientes $(21,4 \%)$ $\mathrm{RP}$ y en tres casos $(21,4 \%)$ no hubo respuesta. Las complicaciones más frecuentes asociadas al uso de quimioterapia fueron infecciosas $(7$ casos, $50 \%)$, con cuatro casos de sepsis de foco pulmonar $(28,5 \%)$.

Al término de este reporte, la mortalidad global de la serie fue de ocho pacientes $(57,1 \%)$, con una mediana de supervivencia de 5,82 meses $(2,58$ 26,19) (Tabla 5). La curva de sobrevida actuarial, para los pacientes de esta serie, se ilustra en la Figura 2. Cinco pacientes (35,7\%) fallecieron por progresión del proceso linfoproliferativo y tres $(21,4 \%)$ lo hicieron a causa de un cuadro séptico secundario.

Existen seis casos sobrevivientes a junio de 2009: un paciente con LNH Plasmablástico de la cavidad oral, tratado con quimioterapia $\mathrm{CHOP} /$ Intratecal en RC luego de 49 meses de sobrevida; ambos casos de LH celularidad mixta, uno de ellos tratado con quimioterapia ABVD asociada a radioterapia, en RC con 30 meses de sobrevida y otro tratado con quimioterapia ABVD exclusiva, con una sobrevida de 25 meses en RC; un paciente con LNH DCGB variante anaplásica, que completó seis ciclos de quimioterapia CHOP-Intratecal, en RC luego de más de 6 meses; un paciente con $\mathrm{LNH}$ Burkitt, tratado con quimioterapia $\mathrm{CHOP}$ intrate-

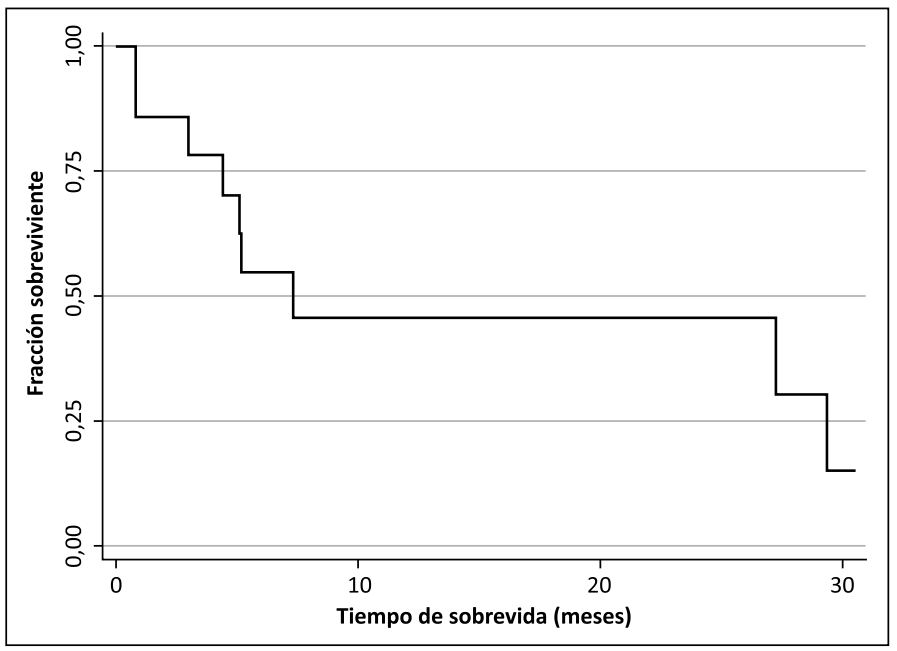

Figura 2. Sobrevida actuarial en pacientes con Linfoma y VIH $(n=22)$. 
cal, en RP con un tiempo de sobrevida de 17 meses y un caso de LNH DCGB tipo centro germinal, diagnosticado hace menos de 2 meses, cursando su segundo ciclo de quimioterapia $\mathrm{CHOP}$.

\section{Discusión}

En este reporte la incidencia acumulada para la asociación diagnóstica Linfoma/VIH es mayor a la descrita en publicaciones nacionales previas ${ }^{8,9}$, pero cercana a los rangos de series internacionales, que oscilan entre $3-10 \%^{2,18}$.

La edad mediana en esta serie fue de 39,5 años, lo cual es concordante con otros reportes ${ }^{7,18-20}$. Por otra parte, existió un claro predominio de pacientes varones en este estudio, hecho que se ha atribuido a una mayor incidencia de infección por VIH en pacientes del género masculino ${ }^{20-23}$.

En todos los casos el diagnóstico de linfoma se efectuó en etapa SIDA, con seis pacientes $(42,8 \%)$ con recuentos de linfocitos CD4 $<50$ céls $/ \mathrm{mm}^{3}$, lo cual es compatible con el incremento del riesgo de ocurrencia de linfoma en estados de inmunodeficiencia severa ${ }^{2,8}$.

La presencia de síntomas B del linfoma y la afectación ganglionar fue superior a la descrita en la literatura, la cual señala presencia de adenopatías en un tercio de los pacientes durante la era pre TARGA y en cerca de $16 \%$ de los casos luego de la instauración de la TARGA ${ }^{1}$. Del mismo modo, destaca el alto porcentaje de pacientes que presentaron compromiso extranodal (50\%), siendo dicho hallazgo semejante a cifras de reportes previos, que oscilan entre 40 y $65 \%{ }^{24,25}$.

Si bien los hallazgos de laboratorio no fueron específicos, algunos elementos como las alteraciones del hemograma, la elevación de la VHS y el ascenso de la LDH en su conjunto, pueden contribuir al diagnóstico de linfoma en estos pacientes. Sin embargo, dichas alteraciones pueden estar dadas exclusivamente por la infección por VIH, aún sin el diagnóstico concomitante de linfoma. Dado que el estudio de $\beta-2$ microglobulinemia no se encuentra disponible de forma rutinaria en el centro hospitalario descrito, no es posible determinar cual fue el comportamiento de esta prueba en este grupo de pacientes, aún cuando su utilidad como herramienta diagnóstica y pronóstica es ampliamente reconocida ${ }^{1}$.

Respecto a los tipos de linfoma, se evidenció un claro predominio de LNH por sobre $\mathrm{LH}$ y, particularmente, de subtipos sistémicos agresivos. Los tipos histológicos más frecuentes, tanto para LNH como para LH, concuerdan con la literatura internacional ${ }^{1,18-19,24-27}$. Es necesario destacar la existencia de dos casos de LNH Plasmablástico, entidad poco frecuente asociada específicamente a infección por VIH, para la cual existe un bajo número de casos descritos ${ }^{28}$.

Dado el número reducido de pacientes en estudio y la variabilidad en los esquemas terapéuticos utilizados, es improcedente pretender estimar el impacto de la terapia antirretroviral en la sobrevida de los casos estudiados. No obstante, destaca el hecho que todos los pacientes sobrevivientes a la fecha, hayan recibido TARGA asociada a quimioterapia intravenosa, y más aún, que cinco de ellos se encuentren en RC a la fecha de redacción de este informe. Dicha observación concuerda con algunos reportes que han demostrado la viabilidad del uso concomitante de quimioterapia y TARGA $22-23,25,29$.

Considerando el grado de compromiso clínico e inmunológico, los tipos histológicos más frecuentes y el bajo número de pacientes que se encontraba recibiendo TARGA al momento del diagnóstico de linfoma, se sugiere que el comportamiento de esta serie respecto a la asociación diagnóstica Linfoma/VIH se asemeja al observado durante la era pre-TARGA, con predominio de aquellos tipos histológicos definitorios de SIDA y bajos porcentajes de remisión completa. Adicionalmente, en esta serie no se reportan casos de LNH primario de SNC, debido a la falta de confirmación histológica para lesiones del SNC.

Si bien la mortalidad global de esta serie fue de ocho pacientes $(57,1 \%)$, es menor a la descrita en reportes nacionales previos ${ }^{12}$. La curva de supervivencia estimada, indica que la probabilidad de supervivencia presenta un quiebre aproximadamente a los ocho meses del diagnóstico de linfoma, siendo menor al $50 \%$ a veinte meses plazo.

Dada la gran variabilidad en los sistemas de clasificación para neoplasias de origen linfoide, resulta imprescindible unificar los criterios de clasificación. En este contexto, la IHQ resulta ser un elemento fundamental cuya implementación no puede obviarse en estudios de esta naturaleza.

El mal pronóstico de la asociación LinfomaVIH y los avances en las alternativas terapéuticas disponibles, ratifican la necesidad de profundizar 
en el estudio de esta temática. Considerando la baja prevalencia de Linfoma en pacientes con SIDA, resulta fundamental contar con estudios multicéntricos que permitan agrupar un mayor número de pacientes con la asociación diagnóstica descrita. No obstante, las experiencias de centros individuales, como es el caso de este reporte, aportan una visión realista de la realidad particular y constituyen el punto de partida para el eventual desarrollo de estudios a mayor escala.

\section{Referencias}

1. Jaffe E, Campo E, Lee N, Swerdlow S, Pileri S. WHO Classification of Tumours of Haematopoietic and Lymphoid Tissue; International Agency for Research on Cancer; 4 Ed; 2008; p. 340-2.

2. Biggar R, Chaturvedi A, Goedert J, Engels E. AIDSRelated Cancer and Severity of Immunosuppression in Persons With AIDS. J Natl Cancer Inst 2007; 99: 962-72.

3. Palmieri C, Triebel T, Large O, Bower M. AIDS-related non-Hodgkin's lymphoma in the first decade of highly active antiretroviral therapy. Q J Med 2006; 99: 811-26.

4. Alexander D, Mink P, Adami H, Chang E, Cole P, Mandel J, Trichopoulos D. The non-Hodgkin lymphomas: A review of the epidemiologic literature. Int J Cancer 2007; 120: 1-39.

5. Mbulaiteye S, Katabira E, Wabinga H, Parkin D, Virgo P, Ochai R, et al. Spectrum of cancers among HIV-infected persons in Africa: The Uganda AIDS-Cancer Registry Match Study. Int J Cancer 2006; 118: 985-90.

6. Mwakigonja A, Kaaya E, Mgaya E. Malignant lymphomas and HIV infection in Tanzania. J Exp Clin Cancer Res 2008, 27: 9.

7. Engels E, Biggar R, Hall H, Cross H, Crutchfield A, Finch J, et al. Cancer risk in people infected with human immunodeficiency virus in the United States. Int J Cancer, 2008. 123: 187-94.

8. Stein L, Urban M, O'Connell D, Qin Yu X, Beral V, Newton R, et al. The spectrum of human immunodeficiency virus-associated cancers in a South African black population: Results from a case control study, 1995-2004. Int J Cancer 2008; 122: 2260-5.

9. Boletín epidemiológico CONASIDA MINSAL Chile, 2004.

10. Wolff M, Northland R, Segovia J, Beltrán C, Valdés M, Albornoz M, et al. Características clínicas e historia natural de la infección por virus de la inmunodeficiencia humana. Estudio en una población chilena atendida en un centro piloto multiprofesional. Rev Med Chile 1995; 123: 61-73.
11. Wolff M, Diomedi A, Morales O, Bidart T, Dabanch J, Bustamante C, et al. Seguimiento prospectivo de una población infectada por VIH con y sin posibilidades de terapia anti-retroviral: impacto en sobrevida y complicaciones. Rev Med Chile 2001; 129: 886-94.

12. Osorio G, Montenegro C. Linfomas asociados a infección por virus de inmunodeficiencia humana en un complejo hospitalario de la Región Metropolitana, Chile: 1990-2002. Reporte de 14 casos y revisión. Rev Chil Infect 2007; 24: 117-24.

13. Rosenberg SA. Validity of the Ann Arbor staging classification for the non-Hodgkin's lymphomas. Cancer Treat Rep 1977; 61: 1023-7.

14. Lister TA, Crowther D, Sutcliffe SB, Glatstein E, Canellos GP, Young RC, et al. Report of a committee convened to discuss the evaluation and staging of patients with Hodgkin's disease: Cotswolds meeting. J Clin Oncol 1989; 7: 1630-6.

15. The International Non-Hodgkin's Lymphoma Prognostic Factors Project: A predictive model for aggressive non-Hodgkin's lymphoma. N Engl J Med 1993; 329: 987-94.

16. Hasenclever D, Volker D. A prognostic score for advanced Hodgkin's disease. International Prognostic Factors Project on Advanced Hodgkin's Disease. N Engl J Med 1998; 339: 1506-14.

17. PANDA. Protocolos Nacionales, Programa de Drogas Antineoplásicas del Adulto 2000- 2001, Unidad de Cáncer, Ministerio de Salud, Chile, p 29.

18. Jacomet C, Lesens O, Villemagne B, Darcha C, Tournilhac O, Henquell C, et al. Non Hodgkin's and Hodgkin's lymphomas and HIV: frequency, outcome and immune response under HAART; Clemont-Ferrand University Hospital 1991- 2003. Med Mal Infect 2006; 36: 157-62.

19. KIM JS, Kim SJ, Kim JS, Kim ES, Shin HJ, Chung JS, et al. Report of AIDS-related lymphoma in South Korea. Jpn J Clin Oncol 2008; 38: 134-9.

20. Sharma A, Bajpai J, Raina V, Mohanti BK. HIV-associated non-Hodgkin's lymphoma: experience from a regional cancer center. Indian J Cancer 2010; 47: 35-9.

21. Wolff M. Cambios epidemiológicos en las enfermedades infecciosas en Chile durante la década 1990-2000. Rev Med Chile 2002; 130: 353-62.

22. Chow KU, Mitrou PS, Geduldig K, Helm EB, Hoelzer D, Brodt HR. Changing incidence and survival in patients with aids-related non-Hodgkin's lymphomas in the era of highly active antiretroviral therapy (HAART). Leuk Lymphoma 2001; 41: 105-16.

23. Diamond C, Taylor TH, Im T, Anton-Culver H. Presentation and outcomes of systemic non-Hodgkin's lymphoma: a comparison between patients with acquired 
immunodeficiency syndrome (AIDS) treated with highly active antiretroviral therapy and patients without AIDS. Leuk Lymphoma 2006; 47: 1822-9.

24. Miralles P, Berenguer J, Ribera J, Calvo F, Mediavilla J, Diez-Martin J, et al. Recomendaciones de GESIDA/ PETHEMA sobre el diagnóstico y el tratamiento de los linfomas en pacientes infectados por el virus de la inmunodeficiencia humana. Med Clin (Barc) 2008; 130: 300-11.

25. Robotin MC, Law MG, Milliken S, Goldstein D, Garsia RJ, Dolan GM, et al. Clinical features and predictors of survival of AIDS- related non-Hodgkin's lymphoma in a population-based case series in Sydney, Australia. HIV
Med 2004; 5: 377-84.

26. Biggar R, Jaffe E, Goedert J, Chaturvedi A, Pfeiffer R, Engels E. Hodgkin lymphoma and immunodeficiency in persons with HIV/AIDS. Blood 2006; 108: 3786-91.

27. Carbone A, Annunziata G, Serraino D, Spina M. HIV associated Hodgkin lymphoma. Current opinion in HIV and AIDS 2009; 4: 3-10.

28. Castillo J, Pantanowitz L, Dezube B. HIV-associated plasmablastic lymphoma: Lessons learned from 112 published cases. Am J Hematol 2008; 83: 804-9.

29. Ribera JM, Navarro JT. Human immunodeficiency virusrelated non-Hodgkin's lymphoma. Haematologica 2008; 93: 1129-32. 